\title{
Detection and Resolution of Terrestrial Gamma Flashes at Telescope Array
}

\author{
Jackson Remington*, Rasha Abbasi, John Belz, Ryan Le Von ${ }^{\dagger}$ \\ Telescope Array Collaboration, University of Utah \\ E-mail: jremingtondcosmic.utah.edu
}

\section{Paul Krehbiel, William Rison, Dan Rodeheffer, Mark Stanley}

Langmuir Labs, New Mexico Institute of Mining and Technology

\begin{abstract}
The Telescope Array Surface Detector (TASD) in western Utah is designed for the measurement of Ultra-High-Energy Cosmic Rays (UHECRs) with characteristically low flux. During its ten years of operation, however, TASD recorded at least 24 bursts of gamma-ray showers, each consisting of two or more TASD events within a single millisecond of lightning activity. After implementation of specialized lightning detectors, the bursts were further correlated with lightning leaders preceding each stroke. We conclude that these bursts are downward Terrestrial Gamma Flashes (TGFs), similar to those observed by satellite. These data constitute the majority of all data on such events recorded from the ground. We are recreating observations using Geant 4 simulations to better understand the properties of downward TGFs and the acceptance statistics of TASD. Acceleration mechanisms, for example, are poorly understood. Spectra from models such as Relativistic Runaway Electron Avalanche (RREA) and Fast Negative Breakdown (FNB) are used to generate a detector response to replicate empirical properties, especially the unique waveform timing and footprints seen at TASD. This also helps characterize the TASD acceptance and aperture for TGFs in a manner similar to cosmic ray studies.
\end{abstract}

36th International Cosmic Ray Conference -ICRC2019-

July 24th - August 1st, 2019

Madison, WI, U.S.A.

\footnotetext{
${ }^{*}$ Speaker.

${ }^{\dagger}$ For collaboration list see PoS(ICRC2019)1177
} 


\section{Discussion}

Terrestrial Gamma Flashes were discovered in 1994 by the orbiting BATSE experiment [1] as a short, intense burst of gamma radiation. Since then, the phenomenon has been studied and identified as originating from the initial, upward breakdown in lightning discharges [2]. Until recently, only a few of these events had been seen from the ground [3] [4] [5], apparently due to the downward analog.

Despite its low lightning frequency, the Telescope Array in western Utah has captured several of these events in the last few years with the addition of some specialized lightning instruments. In 2013, the upgrade began with the Lightning Mapping Array (LMA) [6] to recreate threediemnsional lightning activity above the array, as well as several sferic sensors to record electric field changes. These led to new observations about the nature of TGF production in the leader phase of lightning development [7] [8] (see Figure 1).

Unlike other specialized lightning/TGF experiments, TASDs use plastic scintillators which give much better timing resolution $(\simeq 40 \mathrm{~ns})$ than the typical NaI detectors. In 2018 , TA recieved another lightning upgrade to maximize its advantage; a broadband interferometer (INTF) [9] was installed with much better spatial and temporal resolution than the LMA. Figure 2 Shows an example of INTF data taken during a cloud-to-ground lightning strike.

Results of this state-of-the-art instrumentation upgrade will be presented at this conference.

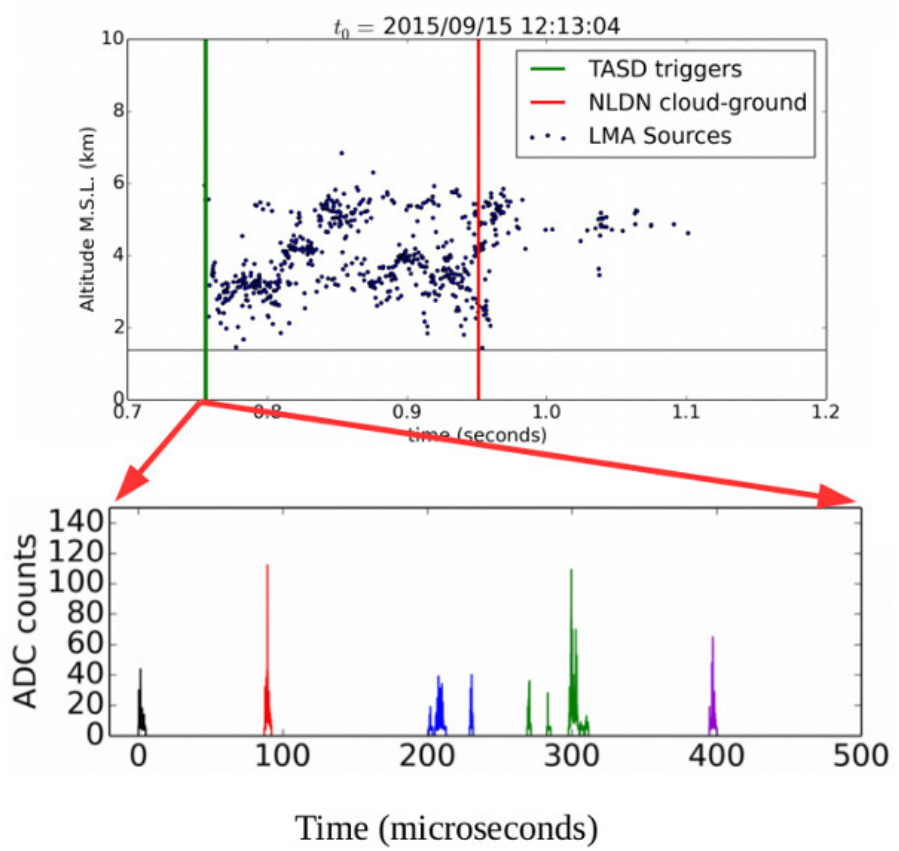

Figure 1: Top: LMA data points recreating a $\simeq 0.4$-second lightning strike. Green line represents TASD activity, red line shows the main ground stroke. Bottom: Energy deposit in a single detector below lightning activity ( $1 \mathrm{MeV}$ of deposited energy $\simeq 18$ ADC counts). 


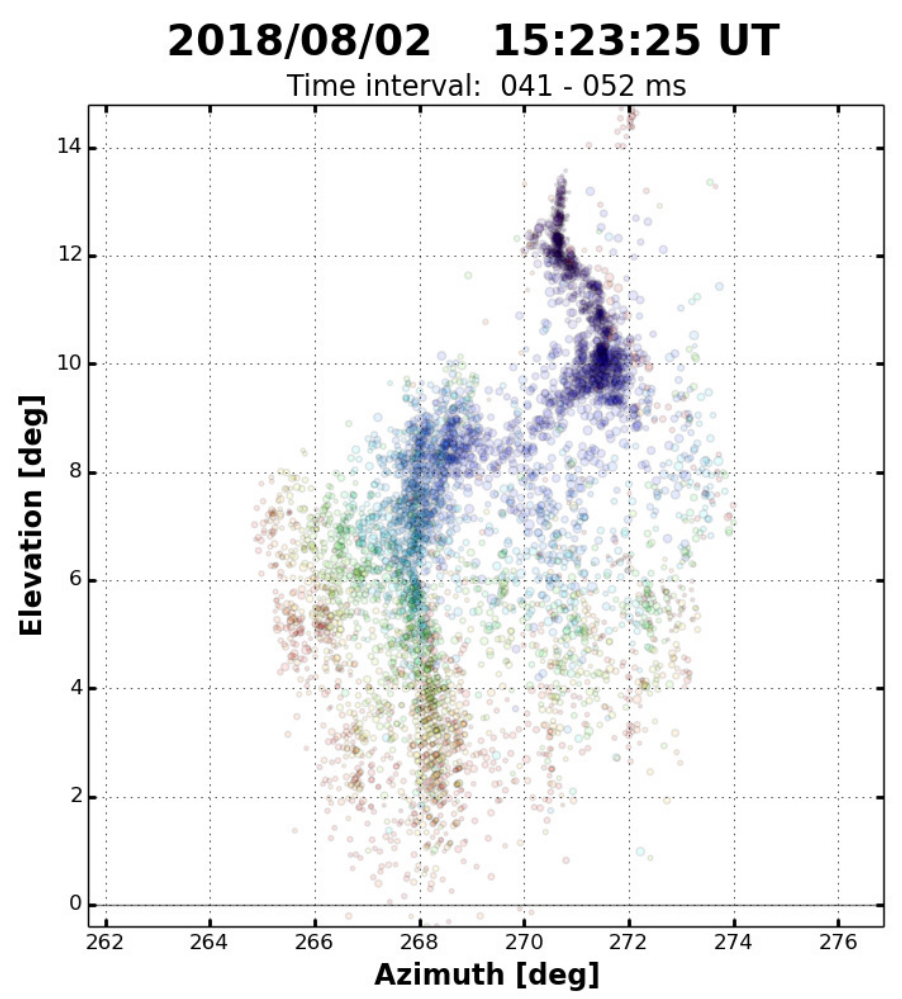

Figure 2: Space-domain plot of INTF data points during the first $11 \mathrm{~ms}$ of a lightning strike over Telescope Array.

\section{Acknowledgements}

The work reported here was partially supported by National Science Foundation grants AGS1205727, AGS-1613260, and AGS-1844306. We thank VAISALA for providing NLDN data under their academic research use policy.

\section{References}

[1] Kouveliotou, C. (1994), The Astrophysical Journal Supplement, 92, 637-642

[2] Cummer, S. A. et al. (2015), Geophys. Res. Lett., 42, 77927798

[3] Dwyer, J. et al. (2012), Journal of Geophysical Research, 117

[4] Tran, M. et al. (2015), Journal of Atmospheric and Solar-Terrestrial Physics, 136

[5] Ringuette, R. et al. (2013), Journal of Geophysical Research: Space Physics, 118

[6] Rison, W., et al. (1999), Geophysical Research Letters, 26(23), 3573-3576.

[7] Abbasi, R., et al. (2017), Physics Letters A, 381(32), 2565 - 2572.

[8] Abbasi, R., et al. (2018), J. Geophys. Res.: Atm. 123 6864-6879 (2018).

[9] Stock, M. G. et al. (2014), J. Geophys. Res. 119 3134â̆̆Ş3165. 